\title{
ON THE TRANSITIVITY OF PERSPECTIVITY IN CONTINUOUS GEOMETRIES*
}

\author{
BY \\ ISRAEL HALPERIN $\dagger$
}

Introduction. The class of finite dimensional projective geometries has been extended to include non-finite dimensional ones by J. von Neumann's remarkable discovery of continuous geometries. In an axiomatic formulation of the geometry as an irreducible complemented modular lattice $\$$ the finiteness of the dimensionality is guaranteed by a chain condition. Von Neumann drops this chain condition and, retaining explicitly only two of its weak consequences, namely, completeness of the geometry and a certain continuity of the lattice operations, succeeds in establishing the existence of an essentially unique real-valued dimension function which may have either a discrete bounded range (the classical finite dimensional projective geometries) or a continuous bounded range (the new continuous geometries). In every case it is understood that the dimension function $D(a)$ is to satisfy

$$
D(a+b)+D(a b)=D(a)+D(b)
$$

for all $a, b$.

It is clear that such a dimension function will be closely connected with perspectivities. For $a, b$ are said to be perspective if there exists a $c$ such that

$$
a+c=b+c, \quad a c=b c ;
$$

and for such $a, b$ (1) implies

$$
\begin{aligned}
D(a)+D(c) & =D(a+c)+D(a c) \\
& =D(b+c)+D(b c)=D(b)+D(c)
\end{aligned}
$$

and hence, if $D(c)$ is finite, $D(a)=D(b)$. This motivates a definition of equidimensionality, namely, $a$ and $b$ are called equidimensional if and only if they are perspective. That this definition will lead to the desired dimension function (in an irreducible system) depends in an essential way on the funda-

* Presented to the Society, December 30, 1936; received by the editors September 14, 1937.

$\dagger$ Sterling Research Fellow.

$\ddagger$ See J. von Neumann: (1) Proceedings of the National Academy of Sciences, vol. 22 (1936), pp. 92-100, 101-108; (2) Lectures on Continuous Geometry, planographed, Institute for Advanced Study, Princeton, N. J., 1935-1937; (3) Continuous Geometry, American Mathematical Society Colloquium Lectures, to appear in book form. (2) will be referred to as C.G. The writer wishes to express his thanks to Professor von Neumann for many discussions of his new geometries.

$\S$ See G. Birkhoff, Annals of Mathematics, vol. 36 (1935), pp. 743-748. 
mental theorem that $a, b$ equidimensional and $b, c$ equidimensional together imply $a, c$ equidimensional; in other words, that the relation of perspectivity is transitive.

The transitivity of perspectivity has been established by von Neumann for reducible as well as irreducible systems* but partly by indirect methods which require the full force of the completeness and continuity axioms. Now while these axioms are indeed necessary for the existence of the dimension function (in irreducible systems), weaker ones will secure the transitivity of perspectivity (in reducible as well as irreducible systems), in fact, just those parts of von Neumann's axioms which involve at most countable sets of elements. $\dagger$

The present paper is devoted chiefly to a proof of the transitivity of perspectivity which uses direct methods throughout and holds for all systems satisfying these weaker axioms. The paper is divided into six sections. The weakened set of axioms to be used is formulated in $\$ 1$. We require parts of C.G., part I, usually in very specialized form, and for convenience these are collected (briefly) in $\$ \S 2,3,4$. The new material in the proof of the transitivity of perspectivity is contained in $\$ 5$. The additivity and continuity properties of perspectivity are established in $\$ 6$. The Lemma 5.1 in $\$ 5$ may perhaps be not without some interest of its own.

1. The partially ordered system. We shall consider a system $L$ of elements $a, b, c, \cdots, x, y, u, v, \cdots, A, B, \cdots$ which is partially ordered, that is, we shall assume that a relation $a \leqq b$ (written equivalently $b \geqq a$ ) holds for certain pairs of elements of $L$ in such a way that

(i) $a \leqq b, b \leqq c$ together imply $a \leqq c$, and

(ii) $a \leqq b, b \leqq a$ are together equivalent to $a=b$.

The following axioms are postulated:

Axiom I. Countable completeness. For every finite or countably infinite set $\ddagger$ of elements $a_{1}, a_{2}, \cdots$ there exist the following elements:

$\mathrm{I}_{1}$. a sum element a (written $\sum_{n} a_{n}$ or equivalently $a_{1}+a_{2}+\cdots$ ) such that for any $x$ of $L, x \geqq a$ if and only if $x \geqq a_{n}$ for every $n$,

$\mathrm{I}_{2}$. an intersection element a (written $\prod_{n} a_{n}$ or equivalently $a_{1} a_{2} \cdots$ ) such that for any $x$ of $L, x \leqq a$ if and only if $x \leqq a_{n}$ for every $n$.

* For the general case see C.G., part III, p. 22, Theorem 2.3; the special (irreducible) case is also a consequence of the theorems of C.G., part I (see C.G., part I, p. 49, corollary to Theorem 5.16).

$\dagger$ That the "countable" axioms are really weaker than the original axioms of von Neumann can be shown by a simple example which satisfies the "countable" axioms but which has no zero, and hence is not complete.

$\ddagger$ All sets considered in this paper will be non-void. Thus Axiom I does not imply the existence of a zero or of a unit element. 
Axiom II. Countable continuity. Let $a_{1}, a_{2}, \cdots$ be any countably infinite sequence, and let $c$ be an arbitrary element of $L$. Then

$\mathrm{II}_{1} .\left(\sum_{n} a_{n}\right) c=\sum_{p}\left\{\left(\sum_{n=1}^{p} a_{n}\right) c\right\}$;

$\mathrm{II}_{2}$. $\left(\prod_{n} a_{n}\right)+c=\prod_{p}\left\{\left(\prod_{n=1}^{p} a_{n}\right)+c\right\}$.

Axiom III. Modularity. For all $a, b, c$,

$$
(a+b) c=\{a+(a+c) b\} c,
$$

or what is equivalent, $a \leqq c$ implies $(a+b) c=a+b c$.

Axiom IV. Complementation. For any three $a, b, c$ such that $a \leqq b \leqq c$ there exists an element $d$ such that $b+d=c, b d=a$.

2. Independent sets of elements. We make the following definition:

Definition 2.1. A finite ( $\geqq 2)$ or countably infinite set of elements $a_{1}, a_{2}, \cdots$ is independent (written $\left(a_{n}, n=1,2, \cdots\right) \perp$ ) if for every two mutually exclusive subsets $a_{i_{1}}, a_{i_{2}}, \cdots$ and $a_{j_{1}}, a_{j_{2}}, \cdots$

$$
\left(\sum_{n} a_{i_{n}}\right)\left(\sum_{n} a_{j_{n}}\right)=\prod_{n} a_{n} .
$$

The $a_{n}$ are said to be independent over $\theta$ if all such $\left(\sum_{n} a_{i_{n}}\right)\left(\sum_{n} a_{j_{n}}\right)$ equal $\theta .^{*}$

LEMMA 2.1. If the $a_{n}$ are independent over $\theta$, then $\theta=\prod_{n} a_{n}$ and $\left(a_{n}\right.$, $n=1,2, \cdots) \perp$.

Proof. Since $\prod_{n} a_{n}=a_{1}\left(\prod_{n \neq 1} a_{n}\right)$, the lemma follows from Definition 2.1.

LEMMA 2.2. If $a_{1}, a_{2}, \cdots$ are independent over $\theta$, then every subset $a_{i_{1}}, a_{i_{2}}, \cdots$ is independent over $\theta$.

Proof. The lemma follows directly from Definition 2.1 and Lemma 2.1.

LEMMA 2.3. If $a_{1}, a_{2}, \cdots$ are independent over $\theta$ and if $\left(a_{i_{r}}, r=1, \cdots\right)$ are mutually exclusive subsets for $i=1,2, \cdots$, then $\sum_{r} a_{i_{r}}, i=1,2, \cdots$, are independent over $\theta$.

Proof. The lemma follows immediately from Definition 2.1 .

LEMMA 2.4. If $\theta, a_{1}, a_{2}, \cdots$ are such that for every two finite and mutually exclusive subsets $a_{i_{1}}, \cdots, a_{i_{p}}$ and $a_{j_{1}}, \cdots, a_{i_{q}}$

$$
\left(\sum_{n=1}^{p} a_{i_{n}}\right)\left(\sum_{n=1}^{q} a_{j_{n}}\right)=\theta
$$

then the $a_{n}$ are independent over $\theta$.

* If $\theta$ is a zero element of $L$, that is, if $a \geqq \theta$ holds for every $a$ in $L$, then our independence over $\theta$ is precisely the notion of independence as used in C.G., part I, chap. 2. 
Proof. Let $a_{i_{n}},(n=1, \cdots)$, and $a_{j_{n}},(n=1), \cdots$, be any two mutually exclusive subsets of $a_{1}, a_{2}, \cdots$. Then

$$
\begin{aligned}
\left(\sum_{n} a_{i_{n}}\right)\left(\sum_{n} a_{j_{n}}\right) & =\left(\sum_{n} a_{i_{n}}\right)\left\{\sum_{q}\left(\sum_{n=1}^{q} a_{i_{n}}\right)\right\} \\
& =\sum_{q}\left\{\left(\sum_{n} a_{i_{n}}\right)\left(\sum_{n=1}^{q} a_{j_{n}}\right)\right\} \\
& =\cdots=\sum_{q}\left\{\sum_{p}\left(\sum_{n=1}^{p} a_{i_{n}}\right)\left(\sum_{n=1}^{q} a_{j_{n}}\right)\right\} \\
& =\sum_{q}\left\{\sum_{p}(\theta)\right\}=\theta
\end{aligned}
$$

and the lemma follows from Definition 2.1.

Corollary. A countably infinite set of elements is independent over $\theta$ if and only if every finite ( $\geqq 2)$ subset is independent over $\theta$.

Proof. The corollary follows immediately from Lemmas 2.2 and 2.4.

Lemma 2.5. Let $\theta, a_{1}, a_{2}, \cdots$ satisfy $a_{n} \geqq \theta$ for every $n$. Let $r_{1}, r_{2}, \cdots, r_{m}$ be distinct integers, and let $S$ be any set of integers not containing $r_{m}$. If $a_{r_{m}} \sum_{n \neq r_{m}} a_{n}=\theta$, then

$$
\left(\sum_{n=1}^{m} a_{r n}\right)\left(\sum_{n \in S} a_{n}\right)=\left(\sum_{n=1}^{m-1} a_{r_{n}}\right)\left(\sum_{n \in S} a_{n}\right) .
$$

Proof.

$$
\begin{aligned}
\left(\sum_{n=1}^{m} a_{r_{n}}\right)\left(\sum_{n \in S} a_{n}\right) & =\left(\sum_{n=1}^{m-1} a_{r_{n}}+a_{r_{m}}\right)\left(\sum_{n \neq r_{m}} a_{n}\right)\left(\sum_{n \in S} a_{n}\right) \\
& =\left\{\left(\sum_{n=1}^{m-1} a_{r_{n}}\right)+a_{r_{m}}\left(\sum_{n \neq r_{m}} a_{n}\right)\right\}\left(\sum_{n \in S} a_{n}\right) \\
& =\left(\sum_{n=1}^{m-1} a_{r_{n}}+\theta\right)\left(\sum_{n \in S} a_{n}\right)=\left(\sum_{n=1}^{m-1} a_{r_{n}}\right)\left(\sum_{n \in S} a_{n}\right),
\end{aligned}
$$

which proves the lemma.

LEMMA 2.6. If $\theta, a_{1}, a_{2}, \cdots$ are such that $a_{n+1}\left(a_{1}+\cdots+a_{n}\right)=\theta$ for every $n=1,2, \cdots$, then the $a_{n}$ are independent over $\theta$.

Proof. By Lemma 2.4 we need only show that

$$
\left(\sum_{n=1}^{p} a_{i_{n}}\right)\left(\sum_{n=1}^{q} a_{i_{n}}\right)=\theta
$$


for all finite $p, q$ and different $i_{1}, \cdots, i_{p} ; j_{1}, \cdots, j_{o}$; and this follows from a finite number of applications of Lemma 2.5.

Corollary. If $\theta, c, a_{1}, a_{2}, \cdots$ are such that

$$
a_{n}\left(a_{n+1}+\cdots+a_{n+p}+c\right)=\theta
$$

for all $n \geqq 1, p \geqq 1$, then the $c, a_{n}$ are independent over $\theta$.

Proof. By Lemma 2.6, $c, a_{n+p}, a_{n+p-1}, \cdots, a_{n}$ are independent over $\theta$ for all $n \geqq 1, p \geqq 0$. Therefore, by the corollary to Lemma $2.4, c, a_{1}, a_{2}, \cdots$ are independent over $\theta$.

LEMma 2.7. Let $a_{1}, a_{2}, \cdots$ be independent over $\theta$. If $S_{1}, S_{2}, \cdots$ are arbitrary subsets of the integers $1,2, \cdots$ and $S$ is the set of the integers common to all $S_{n}$, then

$$
\prod_{t}\left(\sum_{n \in S_{t}} a_{n}\right)=\sum_{n \in S} a_{n}
$$

Proof. Let $T=\left(a_{r_{1}}, a_{r_{2}}, \cdots\right)$ be the set of the integers not in $S$. Then

$$
\begin{aligned}
\prod_{t}\left(\sum_{n \in S t} a_{n}\right) & =\left(\sum_{n \in S} a_{n}+\sum_{n \in T} a_{n}\right) \prod_{t}\left(\sum_{n \in S t} a_{n}\right) \\
& =\sum_{n \in S} a_{n}+\left(\sum_{n \in T} a_{n}\right) \prod_{t}\left(\sum_{n \in S} a_{n}\right) \\
& =\sum_{n \in S} a_{n}+\sum_{m}\left\{\left(\sum_{t=1}^{m} a_{r_{t}}\right) \prod_{t}\left(\sum_{n \in S_{t}} a_{n}\right)\right\} \\
& =\sum_{n \in S} a_{n}+\sum_{m}(\theta)=\sum_{n \in S} a_{n}
\end{aligned}
$$

(by repeated use of Lemma 2.5) as required.

LEMma 2.8. If $a_{n},(n=1,2, \cdots)$, are independent over $\theta$ and $\theta \leqq u_{n} \leqq a_{n}$ for $n=1, \cdots, p$, and if $\theta \leqq v_{n} \leqq a_{n}$ for $n=1, \cdots, q$, then

$$
\left(\sum_{n=1}^{p} u_{n}\right)\left(\sum_{n=1}^{q} v_{n}\right)=\sum_{n=1}^{\min (p, q)}\left(u_{n} v_{n}\right)
$$

Proof.

$$
\begin{aligned}
\left(u_{1}+u_{2}\right)\left(v_{1}+v_{2}\right) & =\left(u_{1}+u_{2}\right)\left(u_{1}+a_{2}\right)\left(a_{1} v_{1}+v_{2}\right) \\
& =\left(u_{1}+u_{2}\right)\left\{v_{2}+v_{1} a_{1}\left(u_{1}+a_{2}\right)\right\} \\
& =\left(u_{1}+u_{2}\right)\left\{v_{2}+v_{1}\left(u_{1}+a_{1} a_{2}\right)\right\}=\left(u_{1}+u_{2}\right)\left(v_{2}+v_{1} u_{1}\right) \\
& =\cdots=\left(u_{1}+u_{2}\right)\left(u_{1} v_{1}+u_{2} v_{2}\right)=u_{1} v_{1}+u_{2} v_{2} .
\end{aligned}
$$

Thus the lemma holds for $p=\dot{q}=2$. But if the lemma holds for all $p=q<m$, 
then it holds for $p=q=m$ too. For

$$
\left(\sum_{n=1}^{m} u_{n}\right)\left(\sum_{n=1}^{m} v_{n}\right)=\left(\sum_{n=1}^{m-1} u_{n}\right)\left(\sum_{n=1}^{m-1} v_{n}\right)+u_{m} v_{m}=\sum_{n=1}^{m}\left(u_{n} v_{n}\right),
$$

since $\theta \leqq \sum_{n-1}^{m-1} u_{n}, \sum_{n-1}^{m-1} v_{n} \leqq \sum_{n-1}^{m-1} a_{n} ; \theta \leqq u_{m}, v_{m} \leqq a_{m}$; and $\sum_{n-1}^{m-1} a_{n}, a_{m}$ are independent over $\theta$. Thus the lemma holds for all $p=q$. If $p \neq q$, say $p<q$, we can set $u_{n}=\theta$ for $p<n \leqq q$ and apply the result just proved for $p=q$.

LEMMA 2.9. If $a_{1}, a_{2}, \cdots$ are independent over $\theta$ and $\theta \leqq u_{n}, v_{n} \leqq a_{n}$; for $n=1,2, \cdots$, then

$$
\left(\sum_{n} u_{n}\right)\left(\sum_{n} v_{n}\right)=\sum_{n}\left(u_{n} v_{n}\right)
$$

Proof.

$$
\begin{aligned}
\left(\sum_{n} u_{n}\right)\left(\sum_{n} v_{n}\right) & =\left\{\sum_{p}\left(\sum_{n=1}^{p} u_{n}\right)\right\}\left\{\sum_{q}\left(\sum_{n=1}^{q} v_{n}\right)\right\} \\
& =\sum_{q}\left\{\sum_{p}\left(\sum_{n=1}^{p} u_{n}\right)\left(\sum_{n=1}^{q} v_{n}\right)\right\} \\
& =\sum_{q}\left\{\sum_{p}\left(\sum_{n=1}^{\min (p, q)}\left(u_{n} v_{n}\right)\right)\right\} \\
& =\sum_{n}\left(u_{n} v_{n}\right)
\end{aligned}
$$

(by Lemma 2.8) as required.

LEMMA 2.10. Let $a_{1}, a_{2}, \cdots$ be independent over $\theta$. If $a_{i} \geqq a_{i j} \geqq \theta$ for all $i, j$, and if the elements $a_{i j}, j=1, \cdots$, are independent over $\theta$ (whenever there are at least two elements in the set) for every $i=1,2, \cdots$, then the set of all $a_{i j},(i, j=1, \cdots)$, is independent over $\theta$.

Proof. If $a_{i_{r} j_{r}},(r=1, \cdots)$, and $a_{k_{s} l_{s}},(s=1, \cdots)$, are mutually exclusive subsets of the $a_{i j}$, then

$$
\begin{aligned}
\left(\sum_{r} a_{i_{r} i_{r}}\right)\left(\sum_{s} a_{k_{s} l_{s}}\right) & =\left\{\sum_{i_{r}}\left(\sum_{i_{r}} a_{i_{r} i_{r}}\right)\right\}\left\{\sum_{k_{s}}\left(\sum_{l_{s}} a_{k_{s} l_{s}}\right)\right\} \\
& =\sum_{n}\left\{\left(\sum_{i_{r}} a_{n i_{r}}\right)\left(\sum_{l_{s}} a_{n l_{s}}\right)\right\} \\
& =\sum_{n}\{\theta\}=\theta
\end{aligned}
$$

(by Lemma 2.9), which proves the lemma. 
LEMMA 2.11. If $\theta \leqq a_{0}$ and if $a_{n}, a_{n}^{\prime}$ are defined for $n=1,2, \cdots$ in such $a$ way that

$$
a_{n-1}=a_{n}+\dot{a}_{n}^{\prime}, \quad a_{n} a_{n}^{\prime}=\theta,
$$

for $n=1,2, \cdots$, then $\left(\prod_{r} a_{r}\right), a_{n}{ }^{\prime},(n=1,2, \cdots)$, are independent over $\theta$ and $a_{0}=\sum_{n} a_{n}{ }^{\prime}+\prod_{r} a_{r}$.

Proof.

$$
\begin{aligned}
& a_{n}^{\prime}\left(a_{n+1}^{\prime}+a_{n+2}^{\prime}+\cdots+a_{n+p}^{\prime}+\prod_{r} a_{r}\right) \\
& \quad=a_{n}^{\prime} a_{n}\left(a_{n+1}^{\prime}+a_{n+2}^{\prime}+\cdots+a_{n+p}^{\prime}+\prod_{r} a_{r}\right)=\theta
\end{aligned}
$$

for all $n, p$. Hence $\left(\prod_{r} a_{r}\right), a_{1}^{\prime}, a_{2}^{\prime}, \cdots$ are independent over $\theta$ by the corollary to Lemma 2.6. Furthermore

$$
\begin{aligned}
a_{0} & =a_{1}^{\prime}+a_{1}=a_{1}^{\prime}+a_{2}^{\prime}+a_{2}=\cdots \\
& =\sum_{n=1}^{r} a_{n}^{\prime}+a_{r}=\sum_{n=1}^{\infty} a_{n}^{\prime}+a_{r},
\end{aligned}
$$

for $r=1,2, \cdots$. Hence

$$
a_{0}=\prod_{r}\left(\sum_{n=1}^{\infty} a_{n}^{\prime}+a_{r}\right)=\sum_{n=1}^{\infty} a_{n}^{\prime}+\prod_{r=1}^{\infty} a_{r}
$$

and the lemma is proved.

3. Perspectivities and perspective mappings. We make the following definition:

Definition 3.1. $a, b$ are perspective (written $a \sim b)$ if there exists an element $c$ such that

(i) $a+c=b+c$,

(ii) $a c=b c$.

Then $c$ is called the axis of the perspectivity.

LEMma 3.1. If $a, b$ are perspective and $\theta \leqq a b$, then there exists an element $d$ such that

(i) $a+d=b+d=a+b$, and

(ii) $a d=b d=\theta$.

Proof. Let $c$ be an axis of perspectivity for $a$ and $b$. Since $\theta \leqq a b$ $\leqq\{c(a+b)+a b\}$, Axiom IV secures the existence of an element $d$ such that

$$
d+a b=c(a+b)+a b, \quad d a b=\theta .
$$


For this $d$ we have

$$
a+d=a+c(a+b)+a b=(a+c)(a+b)=a+b .
$$

Similarly $b+d=a+b$, and (i) holds.

$$
\begin{aligned}
a d & =a d\{c(a+b)+a b\}=d\{a b+a c(a+b)\} \\
& =d a b \\
& =\theta,
\end{aligned}
$$

since $a c(a+b)=a c \leqq a b$. Similarly $b d=\theta$, and (ii) holds. Thus $d$ satisfies the requirements of the lemma.

Definition 3.2. The sublattice of the $x$ satisfying $x \leqq a$ is denoted by $L(a)$. If $a \leqq b$, the sublattice of the $x$ satisfying $a \leqq x \leqq b$ is denoted by $L(a, b)$.*

LEMмA 3.2. If $a, b$ are perspective with axis $c$ and $\theta=a c=b c$, then $a(1,1)$ correspondence between the elements of $L(\theta, a)$ and those of $L(\theta, b)$ which preserves the relation $\leqq i s$ defined by the inverse mappings

$$
\begin{aligned}
& \text { (P) } a_{1} \rightarrow b_{1}=\left(a_{1}+c\right) b \\
& \text { (Q) } b_{1} \rightarrow a_{1}=\left(b_{1}+c\right) a .
\end{aligned}
$$

Proof. If $\theta \leqq x \leqq a$ then under $(P) x \rightarrow(x+c) b$, and under $(Q)$

$$
\begin{aligned}
(x+c) b & \rightarrow\{(x+c) b+c\} a=(x+c)(b+c) a \\
& =\{c+x(b+c)\} a=c a+x(b+c) \\
& =x(a+c)=x .
\end{aligned}
$$

Hence $(Q)$ is inverse to $(P)$. Similarly $(P)$ is inverse to $(Q)$. It follows that the correspondence is $(1,1)$. The invariance of the relation $\leqq$ is clear from the definition of $(P)$ and $(Q)$.

Definition 3.3. The mappings of Lemma 3.2 are called perspective mappings.

LEMMA 3.3. If $a_{1}$ corresponds to $b_{1}$ under a perspective mapping, then $a_{1} \sim b_{1}$.

Proof. Suppose $a_{1}$ corresponds to $b_{1}$ under a perspective mapping of $L(\theta, a)$ on $L(\theta, b)$ with axis $c$. Then $a_{1}$ is perspective to $b_{1}$ with axis $c$, for

$$
\begin{aligned}
a_{1}+c & =\left(b_{1}+c\right) a+c=\left(b_{1}+c\right)(a+c) \\
& =\left(b_{1}+c\right)(b+c)=b_{1}+c, \\
a_{1} c & =a\left(b_{1}+c\right) c=a c=b c=b\left(a_{1}+c\right) c=b_{1} c,
\end{aligned}
$$

and conditions (i) and (ii) therefore hold.

* The Axioms I, II, III, IV hold in $L(a)$ and in $L(a, b) . L(a)$ has a unit (greatest) element, namely $a$, and $L(a, b)$ has a unit element $b$ and a zero (smallest) element $a$. 
LeMMA 3.4. If $P_{i}$ is a perspective mapping of $L\left(\theta, a_{i}\right)$ on $L\left(\theta, b_{i}\right)$ for $i=1, \cdots, p$, where $a_{i+1}=b_{i}$ for $i=1, \cdots, p-1$, then the product mapping of the $P_{i}$ is $a(1,1)$ mapping of $L\left(\theta, a_{1}\right)$ on $L\left(\theta, b_{p}\right)$ which preserves the relation $\leqq$.

Proof. The lemma follows immediately from Definition 3.3.

Definition 3.4. The mapping of Lemma 3.4 is called a projective mapping.

4. Transitivity of perspectivity in special cases. We prove the following lemma:

LemMA 4.1. $a \sim b, b \sim c,(a, b, c) \perp$ together imply $a \sim c$.

Proof. By Lemma $3.1 x, y$ exist such that

$$
\begin{aligned}
a+x & =b+x=a+b, \quad b+y=c+y=b+c, \\
a x & =b x=\theta, \quad b y=c y=\theta,
\end{aligned}
$$

where $\theta=a b c$.

Then $a$ is perspective to $c$ with axis $d=(a+c)(x+y)$. For

$$
\begin{aligned}
a+d & =a+(a+c)(x+y)=(a+c)(a+x+y) \\
& =(a+c)(a+b+y)=(a+c)(a+b+c)=a+c .
\end{aligned}
$$

Similarly $c+d=a+c$. Thus $a+d=c+d$, and (i) holds. Also

$$
\begin{aligned}
a d & =a(a+c)(x+y)=a(x+y)=a(a+b)(x+y) \\
& =a\{x+(a+b) y(b+c)\}=a\{x+\theta\}=\theta .
\end{aligned}
$$

Similarly $c d=\theta$. Thus $a d=c d$, and (ii) holds.

Lemma 4.2. $a_{n} \sim b_{n}$, for $n=1,2, \cdots$, and $\left(a_{n}+b_{n}, n=1,2, \cdots\right) \perp$ together imply $\sum_{n} a_{n} \sim \sum_{n} b_{n}$.

Proof. By Lemma 3.1 we may assume the existence of elements $x_{n}$ such that

$$
\begin{aligned}
a_{n}+x_{n} & =b_{n}+x_{n}=a_{n}+b_{n}, \\
a_{n} x_{n} & =b_{n} x_{n}=\theta,
\end{aligned}
$$

where $\theta=\prod_{n}\left(a_{n} b_{n}\right)$. Then $\sum_{n} a_{n}, \sum_{n} b_{n}$ are perspective with axis $\sum_{n} x_{n}$, for the relations

$$
\begin{aligned}
\sum_{n} a_{n}+\sum_{n} x_{n} & =\sum_{n}\left(a_{n}+x_{n}\right) \\
& =\sum_{n}\left(b_{n}+x_{n}\right)=\sum_{n} b_{n}+\sum_{n} x_{n},
\end{aligned}
$$

give property (i); and 


$$
\begin{aligned}
\left(\sum_{n} a_{n}\right)\left(\sum_{n} x_{n}\right) & =\sum_{n}\left(a_{n} x_{n}\right) \\
& =\sum_{n}\left(b_{n} x_{n}\right)=\left(\sum_{n} b_{n}\right)\left(\sum_{n} x_{n}\right)
\end{aligned}
$$

(by Lemma 2.9) gives property (ii).

LEMMA 4.3. If an infinite independent sequence of elements $a_{0}, a_{1}, \cdots$ satisfy $a_{n} \sim a_{n+1}$, for $n=0,1, \cdots$, then $a_{0}=\prod_{n} a_{n}$.

Proof. From Lemmas 2.2 and 4.1, $a_{0} \sim a_{n}$ for all $n$. By Lemma 3.1 we may therefore assume the existence of elements $x_{n},(n=1,2, \cdots)$, such that

$$
\begin{aligned}
a_{0}+x_{n} & =a_{n}+x_{n}=a_{0}+a_{n}, \\
a_{0} x_{n} & =a_{n} x_{n}=\theta,
\end{aligned}
$$

where $\theta=\prod_{n} a_{n}$. We deduce successively

$$
\begin{array}{rlr}
a_{0} & \leqq a_{n}+x_{n}, & n=1,2, \cdots, \\
a_{0} & \leqq\left(\sum_{n=p}^{\infty} a_{n}\right)+\left(\sum_{n=1}^{\infty} x_{n}\right), & p=1,2, \cdots, \\
a_{0} & \leqq \prod_{p}\left\{\left(\sum_{n=p}^{\infty} a_{n}\right)+\left(\sum_{n=1}^{\infty} x_{n}\right)\right\}=\left\{\prod_{p}\left(\sum_{n=p}^{\infty} a_{n}\right)\right\}+\left(\sum_{n=1}^{\infty} x_{n}\right) \\
& =\sum_{n=1}^{\infty} x_{n}
\end{array}
$$

by Lemma 2.7. Hence

$$
a_{0}=a_{0} \sum_{n=1}^{\infty} x_{n}=a_{0}\left(\sum_{p} \sum_{n=1}^{p} x_{n}\right)=\sum_{p}\left(a_{0} \sum_{n=1}^{p} x_{n}\right)=\theta,
$$

if only $a_{0} \sum_{n=1}^{p} x_{n}=\theta$ for $p=1,2, \cdots$. Now for any fixed $p$,

and, since

$$
a_{0} \sum_{n=1}^{p} x_{n}=a_{0}\left\{\sum_{n=1}^{p-1} x_{n}+\left(a_{0}+\sum_{n=1}^{p-1} x_{n}\right) x_{p}\right\}
$$

$$
x_{p}\left(a_{0}+\sum_{n=1}^{p-1} x_{n}\right)=x_{p}\left(a_{0}+a_{p}\right)\left(a_{0}+\sum_{n=1}^{p-1} a_{n}\right)\left(a_{0}+\sum_{n=1}^{p-1} x_{n}\right)=x_{p} a_{0}=\theta,
$$

therefore

$$
a_{0} \sum_{n=1}^{p} x_{n}=a_{0} \sum_{n=1}^{p-1} x_{n}
$$

A finite number of such reductions gives 


$$
a_{0} \sum_{n=1}^{p} x_{n}=a_{0} x_{1}=\theta
$$

as required, and the lemma is proved.

LEMMA 4.4. $a \sim x, x \sim a_{1}, a x \leqq a_{1} \leqq a$ together imply $a_{1}=a$.

Proof. Let $a x=a_{1} x=\theta$. Since $\theta \leqq a_{1} \leqq a$, Axiom IV secures the existence of an element $a_{1}^{\prime}$ such that

$$
a_{1}+a_{1}^{\prime}=a, \quad a_{1} a_{1}^{\prime}=\theta .
$$

By Lemmas 3.1 and 3.2 there exist perspective mappings $T_{1}$ of $L(\theta, a)$ on $L(\theta, x)$ and $T_{2}$ of $L(\theta, x)$ on $L\left(\theta, a_{1}\right)$. Define by induction on $n$

$$
x_{n}=T_{1}\left(a_{n}\right), \quad x_{n}^{\prime}=T_{1}\left(a_{n}^{\prime}\right), \quad a_{n+1}=T_{2}\left(x_{n}\right), \quad a_{n+1}^{\prime}=T_{2}\left(x_{n}^{\prime}\right) .
$$

Then Lemma 2.11, the relation $a x=\theta$, and Lemma 2.10, give $\left(a_{n}{ }^{\prime}, x_{n}{ }^{\prime}\right.$, $n=1,2, \cdots) \perp$. Lemma 2.2 then gives $\left(a_{n}^{\prime}, x_{n}^{\prime}, a_{n+1}^{\prime}\right) \perp$, and Lemmas 3.3 and 4.1 give $a_{n}^{\prime} \sim a_{n+1}^{\prime}$, for $n=1,2, \cdots$. Lemma 2.2 shows that $\left(a_{n}^{\prime}\right.$, $n=1,2, \cdots) \perp$; hence by Lemma $4.3 a_{1}^{\prime}=\prod_{n} a_{n}{ }^{\prime}=\theta$. Thus $a_{1}=a_{1}+\theta$ $=a_{1}+a_{1}^{\prime}=a$; and the lemma is proved.

DefintTION 4.1. If $\theta$ has been defined, we sometimes write

$$
\sum_{n}\left(\oplus x_{n}\right)
$$

(or the equivalent $\left.x_{1} \oplus x_{2} \oplus \cdots\right)$ in place of $\sum_{n} x_{n}$ (or $\left.x_{1}+x_{2}+\cdots\right)$, provided the $x_{n}$ are independent over $\theta$. If $\theta \leqq u \leqq v$, then [v-u] will denote an element (fixed) such that $u \oplus[v-u]=v$. (Such an element exists by Axiom IV.)

Lemma 4.5. $a \sim x, x \sim b, a b \leqq x$ together imply $a \sim b$.

Proof. $(\alpha)$ Consider the special case where $a b=b x=a x$ and $x \leqq a+b$. Let $b_{1}=b(a+x)$. Then $b_{1}$ is perspective to $x$ with axis $a$, for

$$
b_{1}+a=b(a+x)+a=(b+a)(a+x)=x+a,
$$

hence relation (i) holds; and

$$
b_{1} a=b(a+x) a=b a=x a,
$$

hence (ii) holds.

Since $b \sim x$ and $b_{1} x=b(a+x) x=b x, b x \leqq b_{1} \leqq b$; and Lemma 4.4 gives $b_{1}=b$. Hence $b \leqq a+x$. Similarly $a \leqq b+x$. It follows that $a$ is perspective to $b$ with axis $x$, for 


$$
a+x=a+x+b=b+x ;
$$

hence relation (i) holds, and $a x=b x$ (by the special hypotheses of $(\alpha)$ ) hence relation (ii) holds. This proves Lemma 4.5 in the special case $(\alpha)$.

$(\beta)$ Suppose now only that $a b=b x=a x$ (equal, say $\theta$ ). Let $T_{1}, T_{2}$ be perspective mappings of $L(\theta, x)$ on $L(\theta, a)$ and on $L(\theta, b)$, respectively. Set $a_{0}=a$, $x_{0}=x, b_{0}=b$, and define $a_{n}, a_{n}{ }^{\prime}, x_{n}, x_{n}{ }^{\prime}, b_{n}, b_{n}{ }^{\prime}$, for $n=1,2, \cdots$, by induction on $n$ as follows:

$$
\begin{aligned}
x_{n} & =x_{n-1}\left(a_{n-1}+b_{n-1}\right), & a_{n} & =T_{1}\left(x_{n}\right), \\
x_{n}^{\prime} & =\left[x_{n-1}-x_{n}\right], & b_{n} & =T_{2}\left(x_{n}\right), \\
& =T_{1}\left(x_{n}^{\prime}\right), & b_{n}^{\prime} & =T_{2}\left(x_{n}^{\prime}\right) .
\end{aligned}
$$

If we set $\bar{a}=\prod_{n} a_{n}, \bar{x}=\prod_{n} x_{n}, b=\prod_{n} b_{n}$, then Lemma 2.11, the relation $a b=\theta$, and Lemma 2.3 give $a=\bar{a}+\sum_{n} a_{n}{ }^{\prime}, \quad b=\bar{b}+\sum_{n} b_{n}{ }^{\prime}$; and $\bar{a}+\bar{b}, a_{n}{ }^{\prime}+b_{n}{ }^{\prime}$, $(n=1,2, \cdots)$, are independent over $\theta$. Lemma 3.3 shows that $a_{n}{ }^{\prime} \sim x_{n}{ }^{\prime}$ and $x_{n}{ }^{\prime} \sim b_{n}{ }^{\prime}$. Since $a_{n}{ }^{\prime} b_{n}{ }^{\prime}=\theta$ and $x_{n}{ }^{\prime}\left(a_{n}{ }^{\prime}+b_{n}{ }^{\prime}\right)=\theta$, Lemmas 2.6 and 4.1 show that $a_{n}{ }^{\prime} \sim b_{n}{ }^{\prime}$, for $n=1,2, \cdots$. Now Lemma 4.2 shows that $a \sim b$ if only $\bar{a} \sim b$.

$\bar{a}, \bar{x}, b$ satisfy the hypotheses of Lemma 4.5 and the special conditions of $(\alpha)$, for $\bar{a}=T_{1}(\bar{x}), \bar{b}=T_{2}(\bar{x})$ imply $\bar{a} \sim \bar{x}, \bar{x} \sim \bar{b} ; \bar{a} \bar{x}=\bar{a} a x \bar{x}=\theta, b \bar{x}=b b x \bar{x}=\theta$, $\bar{a} \bar{b}=\bar{a} a b \bar{a}=\theta ;$ and, since $x_{n} \leqq a_{n-1}+b_{n-1}$ for all $n$,

$$
\begin{aligned}
\bar{x} & \leqq \prod_{n}\left(a_{n}+b_{n}\right)=\prod_{q} \prod_{p}\left(\prod_{n=1}^{p} a_{n}+\prod_{n=1}^{q} b_{n}\right) \\
& =\prod_{n} a_{n}+\prod_{n} b_{n}=\bar{a}+b
\end{aligned}
$$

by two applications of Axiom $\mathrm{II}_{2}$. Hence $\bar{a} \sim \bar{b}$; and Lemma 4.5 is proved for the special case $(\beta)$.

$(\gamma)$ Suppose now only $a b=b x$ (equal, say $\theta$ ). Let $T_{1}, T_{2}$ be perspective mappings of $L(\theta, a)$ on $L(\theta, x)$ and of $L(\theta, x)$ on $L(\theta, b)$, respectively. Set

$$
\begin{array}{lll}
a_{1}=a x, & x_{1}=T_{1}\left(a_{1}\right)=a_{1}, & b_{1}=T_{2}\left(x_{1}\right), \\
a_{2}=[a-a x], & x_{2}=T_{1}\left(a_{2}\right), & b_{2}=T_{2}\left(x_{2}\right) .
\end{array}
$$

Then $a=a_{1} \oplus a_{2}, x=x_{1} \oplus x_{2}, b=b_{1} \oplus b_{2}$. By Lemma 3.3, $a_{1}=x_{1} \sim b_{1}$. Since the hypotheses of Lemma 4.5 and the special conditions of $(\beta)$ are clearly satisfied by $a_{2}, x_{2}, b_{2}, a_{2} \sim b_{2}$. Lemma 4.2 now gives $a \sim b$, and Lemma 4.5 is established for the special case $(\gamma)$.

( $\delta$ ) Suppose finally only the hypotheses of Lemma 4.5. The method by which $(\gamma)$ was deduced from $(\beta)$ can be applied in the same way to deduce $(\delta)$ from $(\gamma)$. Thus Lemma 4.5 is proved.

CoRollary. If $T_{1}, T_{2}$ are perspective mappings of $L(\theta, a)$ on $L(\theta, x)$, and of $L(\theta, x)$ on $L(\theta, b)$, respectively, and if $T_{2} T_{1}$ maps $a_{1}$ on $b_{1}$, then $a_{1} b_{1}=\theta$ implies $a_{1} \sim b_{1}$. 
Proof. Set $x_{1}=T_{1}\left(a_{1}\right)$. Lemmas 3.3 and 4.5 applied to $a_{1}, x_{1}, b_{1}$, give the desired result.

5. Transitivity of perspectivity. We prove the following lemma:

LEMMA 5.1. If $a_{1} \geqq a_{2} \geqq \cdots$ and $c$ are given, and if $\theta=\prod_{n}\left(a_{n} c\right)$, then there exist decompositions

$$
a_{n}=a_{n} c \oplus a_{n}^{\prime},
$$

for $n=1,2, \cdots$, such that $a_{1}^{\prime} \geqq a_{2}^{\prime} \geqq \cdots \cdot$.

Proof. Let $I_{n}=\left[a_{n}-\left(a_{n} c+a_{n+1}\right)\right]$, for $n=1,2, \cdots$, and let $\bar{a}=\prod_{n} a_{n}$, $I=[\bar{a}-\bar{a} c]$. Then

$$
\begin{aligned}
& a_{1}=I_{1} \oplus\left(a_{1} c+a_{2}\right), \\
& a_{2}=I_{2} \oplus\left(a_{2} c+a_{3}\right), \\
& \cdot \cdot \cdot \cdot \cdot \cdot \cdot \cdot, \\
& a_{n}=I_{n} \oplus\left(a_{n} c+a_{n+1}\right), \\
& \cdot \cdot \cdot \cdot \cdot \cdot \cdot \cdot, \\
& \bar{a}=I \oplus \bar{a} c ;
\end{aligned}
$$

and clearly $c \bar{I}=c \bar{I} \bar{a} c=\theta, c I_{r}=c I_{r}\left(a_{r} c+a_{r+1}\right)=\theta, I_{r+k} \leqq a_{r+k} \leqq a_{r}$, for all $r \geqq 1$, $k \geqq 0$.

We can now prove that $a_{r}=a_{r} c \oplus I \oplus \sum_{n=r}^{\infty} I_{n}$, for $r=1,2, \cdots$. In the first place, $a_{r} c, I, I_{n},(n=r, r+1, \cdots)$ are independent over $\theta$ by the corollary to Lemma 2.6 since $a_{r} c \bar{I}=\theta$; and for all $p>n \geqq r$,

$$
\begin{aligned}
I_{n}\left(a_{r} c+\bar{I}+\sum_{m=n+1}^{p} I_{m}\right) & =I_{n} a_{n}\left(a_{r} c+a_{n+1}\right)\left(a_{r} c+I+\sum_{m=n+1}^{p} I_{m}\right) \\
& =I_{n}\left(a_{n} c+a_{n+1}\right)\left(a_{r} c+I+\sum_{m=n+1}^{p} I_{m}\right) \\
& =\theta .
\end{aligned}
$$

Secondly,

$$
\begin{aligned}
a_{r} c+I+\sum_{n=r}^{\infty} I_{n} & =a_{r} c+\prod_{m=r}^{\infty} a_{m}+\sum_{n=r}^{\infty} I_{n} \\
& =\prod_{m=r}^{\infty}\left(a_{r} c+a_{m}+\sum_{n=r}^{\infty} I_{n}\right) \\
& =\prod_{m=r}^{\infty}\left(a_{r} c+a_{m}+\sum_{n=r}^{m-1} I_{n}\right) .
\end{aligned}
$$

Now if $m \geqq r$, 


$$
\begin{aligned}
a_{r} c+a_{m}+\sum_{n=r}^{m-1} I_{n} & =a_{r} c+a_{m-1} c+a_{m}+I_{m-1}+\sum_{n=r}^{m-2} I_{n} \\
& =a_{r} c+a_{m-1}+\sum_{n=r}^{m-2} I_{n}=\cdots \\
& =a_{r} c+a_{r}=a_{r} .
\end{aligned}
$$

Thus $a_{r} c \oplus I \oplus \sum_{n=r}^{\infty} I_{n}=\prod_{m=r}^{\infty}\left(a_{r}\right)=a_{r}$ as required. It is now clear that if we set

$$
a_{n}^{\prime}=I \oplus \sum_{r=n}^{\infty} I_{r},
$$

we will obtain the decompositions required by the lemma.

CoRollaRy. In Lemma 5.1, $I, I_{n},(n=1,2, \cdots)$, are independent over $\theta$.

Proof. This is immediate from the proof of Lemma 5.1.

Theorem 5.1. Transitivity of Perspectivity. $a \sim x, x \sim b$ together imply $a \sim b$.

Proof. (I) Let $\theta=a b x$, and let $T_{1}, T_{2}$ be perspective mappings of $L(\theta, a)$ on $L(\theta, x)$ and of $L(\theta, x)$ on $L(\theta, b)$, respectively. Let $T=T_{2} T_{1}$ be the product mapping of $L(\theta, a)$ on $L(\theta, b)$, and let $T^{-1}$ be the inverse mapping to $T$. We shall use the notation $a_{1} \rightarrow b_{1}$, or the equivalent $b_{1}=T\left(a_{1}\right)$, to denote that $b_{1}$ is the map of $a_{1}$ under $T$.

(II) Let $c=a b$, and let $a_{0}=[a-c]$, where $a_{0}$ is restricted to satisfy a certain condition which will be stated precisely later (see (III) below). Set $b_{1}=\left(T\left(a_{0}\right)\right) c, b_{1}^{\prime}=\left[T\left(a_{0}\right)-b_{1}\right], a_{1}=T^{-1}\left(b_{1}\right), a_{1}^{\prime}=T^{-1}\left(b_{1}{ }^{\prime}\right)$. Then

$$
\begin{aligned}
a & =a_{0} \oplus c, \\
a_{0}=a_{1} \oplus a_{1}^{\prime}, \quad a_{1} & \rightarrow b_{1} \leqq c, \quad a_{1}^{\prime} \rightarrow b_{1}^{\prime}, \quad\left(b_{1}^{\prime} c=\theta\right) .
\end{aligned}
$$

Since $\theta \leqq b_{1} \leqq a, T\left(b_{1}\right)$ is defined. Let $\left\{T\left(b_{1}\right)\right\} c=b_{2}, b_{2}^{\prime}=\left[T\left(b_{1}\right)-b_{2}\right], b_{12}$ $=T^{-1}\left(b_{2}\right), a_{2}=T^{-1}\left(b_{12}\right), b_{12}^{\prime}=T^{-1}\left(b_{2}^{\prime}\right), a_{2}^{\prime}=T^{-1}\left(b_{12}^{\prime}\right)$. Then

$$
a_{1}=a_{2} \oplus a_{2}^{\prime}, \quad a_{2} \rightarrow b_{12} \rightarrow b_{2} \leqq c, \quad a_{2}^{\prime} \rightarrow b_{12}^{\prime} \rightarrow b_{2}^{\prime}, \quad\left(b_{2}^{\prime} c=\theta\right) .
$$

Similarly, obtain the table

$$
\begin{aligned}
& a_{0}=a_{1} \oplus a_{1}^{\prime}, \quad a_{1} \rightarrow b_{1} \leqq c, \quad a_{1}^{\prime} \rightarrow b_{1}^{\prime}, \quad\left(b_{1}^{\prime} c=\theta\right), \\
& a_{1}=a_{2} \oplus a_{2}^{\prime}, \quad a_{2} \rightarrow b_{12} \rightarrow b_{2} \leqq c, \quad a_{2}^{\prime} \rightarrow b_{12}^{\prime} \rightarrow b_{2}^{\prime}, \quad\left(b_{2}^{\prime} c=\theta\right), \\
& a_{n-1}=a_{n} \oplus a_{n}^{\prime}, \quad a_{n} \rightarrow b_{12} \cdots_{n} \rightarrow b_{23} \cdots_{n} \rightarrow \cdots \rightarrow b_{n} \leqq c, \\
& a_{n}^{\prime} \rightarrow b_{12}^{\prime} \cdots_{n} \rightarrow b_{23}^{\prime} \cdots_{n} \rightarrow \cdots \rightarrow b_{n}^{\prime}, \quad\left(b_{n}^{\prime} c=\theta\right),
\end{aligned}
$$


We can now prove the following statements:

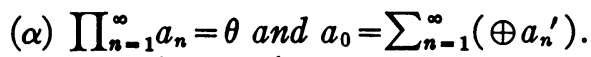

(B) $a_{n}^{\prime}, b_{12}^{\prime} \ldots n, b_{23}^{\prime} \ldots n, \cdots, b_{n}^{\prime}$ are independent over $\theta$, for $n=1,2, \cdots$.

$(\gamma)$ If we set $d_{n}=a_{n}{ }^{\prime}+b_{12}^{\prime} \ldots n+b_{23}^{\prime} \ldots n+\cdots+b_{n}{ }^{\prime}$, then $d_{1}, d_{2}, \cdots$ are independent over $\theta$.

( $\delta$ ) All the primed elements $a_{1}^{\prime}, a_{2}^{\prime}, \cdots, b_{1}{ }^{\prime}, b_{12}^{\prime}, \cdots$ are independent over $\theta$.

Proof of $(\alpha)$. Let $\prod_{n} a_{n}=\bar{a}$. Then $T^{n}(\bar{a})=T T \cdots T(\bar{a})(n$ factors $T)$ is defined for $n=1,2, \cdots$; and $T(\bar{a}), T^{2}(\bar{a}), \cdots$ are independent over $\theta$ by the corollary to Lemma 2.6 since

$$
\left\{T^{n}(\bar{a})\right\}\left\{T^{n+1}(\bar{a})+\cdots+T^{n+p}(\bar{a})\right\}
$$

has a $T^{-n}$ map which is $\leqq a_{0} b=a_{0} a b=a_{0} c=\theta$, for all $n, p \geqq 1$. Since $T^{n}(\bar{a})$ $\sim T^{n+1}(\bar{a})$ by Lemma 3.3, Lemma 4.3 shows that $\bar{a}=\theta$. The statement $(\alpha)$ now follows from Lemma 2.11.

Proof of $(\beta)$.

$$
b_{r(r+1)}^{\prime} \ldots n\left(b_{(r+1)(r+2) \ldots n}^{\prime}+b_{(r+2)(r+3)}^{\prime} \ldots n+\cdots+b_{n}^{\prime}\right)=\theta
$$

since it has a $T^{-r}$ map which is $\leqq a_{0} b=\theta$. The statement $(\beta)$ now follows from the corollary to Lemma 2.6.

Proof of $(\gamma) . \theta \leqq d_{n}\left(d_{n+1}+d_{n+2}+\cdots+d_{n+p}\right)$

$$
\begin{aligned}
& \leqq\left(a_{n}^{\prime}+b_{12 \cdots n}^{\prime}+\cdots+b_{n}^{\prime}\right)\left\{\begin{array}{c}
a_{n+1}^{\prime}+b_{12}^{\prime} \cdots(n+1)+\cdots+b_{n+1}^{\prime} \\
+a_{n+2}^{\prime}+b_{12}^{\prime} \cdots\left(n+\cdots+\cdots+b_{n+2}^{\prime}\right. \\
+\cdots \cdots \cdots \cdot \cdots \cdot \cdots \\
+a_{n+p}^{\prime}+b_{12}^{\prime} \cdots(n+p)+\cdots+b_{n+p}^{\prime}
\end{array}\right\} \\
& =a_{n}^{\prime}\left(a_{n+1}^{\prime}+a_{n+2}^{\prime}+\cdots+a_{n+p}^{\prime}\right)+\left(b_{12}^{\prime} \cdots n+\cdots+b_{n}^{\prime}\right)\left\{\begin{array}{c}
b_{12}^{\prime} \cdots(n+1)+\cdots+b_{n+1}^{\prime} \\
+b_{12}^{\prime} \cdots(n+2)+\cdots+b_{n+2}^{\prime} \\
+\cdots \cdots \cdots \cdot \cdot \\
+b_{12}^{\prime} \cdots(n+p)+\cdots+b_{n+p}^{\prime}
\end{array}\right\} \\
& =\theta+T\left(a_{n}+b_{12 \ldots n}+\cdots+b_{(n-1) n)}\left(\begin{array}{c}
a_{n+1}^{\prime}+b_{12}^{\prime} \cdots(n+1)+\cdots+b_{n(n+1)}^{\prime} \\
+a_{n+2}^{\prime}+b_{12}^{\prime} \cdots(n+2)+\cdots+b_{(n+1)(n+2)}^{\prime} \\
+\cdots \cdots \cdots \cdots \cdots+\cdots \\
+a_{n+p}^{\prime}+b_{12}^{\prime} \cdots(n+p)+\cdots+b_{(n+p-1)(n+p)}^{\prime}
\end{array}\right\}\right) \\
& \leqq \cdots \leqq T^{n+1}(\theta)=\theta .
\end{aligned}
$$

The statement $(\gamma)$ now follows from the corollary to Lemma 2.6.

Proof of $(\delta)$. The statement $(\delta)$ follows from $(\beta)$ and $(\gamma)$ by Lemma 2.10.

(III) By the corollary to Lemma $4.5 a_{n}^{\prime} \sim b_{12}^{\prime} \ldots n, b_{12}^{\prime} \ldots n \sim b_{23}^{\prime} \ldots n, \cdots$, $b_{(n-1) n} \sim b_{n}{ }^{\prime}$. Since $a_{n}{ }^{\prime}, b_{12}^{\prime} \ldots n, \cdots, b_{n}{ }^{\prime}$ are independent, repeated application 
of Lemma 4.1 shows that $a_{n}{ }^{\prime} \sim b_{n}{ }^{\prime}$ for $n=1,2, \cdots$. Now set

$$
\begin{aligned}
& A=\sum_{n=1}^{\infty}\left(\oplus a_{n}^{\prime}\right) \oplus \sum_{r \neq s}\left(\oplus b_{r(r+1)}^{\prime} \ldots s\right), \\
& B=\sum_{n=1}^{\infty}\left(\oplus b_{n}^{\prime}\right) \oplus \sum_{r \neq s}\left(\oplus b_{r(r+1)}^{\prime} \ldots s\right) .
\end{aligned}
$$

By Lemma 4.2, $A \sim B$. Furthermore $T(A)=B$.

Now suppose that $a_{0}$ was chosen (in (II) above) in such a way that, the $b_{n}^{\prime}$ having been defined as above, we should have $\left(\sum_{n=1}^{\infty} b_{n}{ }^{\prime}\right) c=\theta$ (that such an $a_{0}$ exists will be shown in (V) below). Setting

$$
g=\left[c-\sum_{r \neq s}\left(\oplus b_{r(r+1)}^{\prime} \ldots s\right)\right]
$$

we have $B g=B g c=g \sum_{r \neq s}\left(\oplus b_{r(r+1)}^{\prime} \ldots s\right)=\theta$, and we can define

$$
h=[b-(B \oplus g)] \text {. }
$$

Then we clearly have

$$
\begin{aligned}
& c=\sum_{r \neq s}\left(\oplus b_{r(r+1)}^{\prime} \ldots s\right) \oplus g, \\
& a=A \oplus g, \\
& b=B \oplus g \oplus h .
\end{aligned}
$$

By Lemma 4.2, $a \sim b$ if only $h=\theta$.

(IV) We proceed to show that $h=\theta$. Let $g^{\prime}=T^{-1}(g+h)$. Since

$$
A \oplus g=a=T^{-1}(b)=T^{-1}(B \oplus g \oplus h)=A \oplus g^{\prime},
$$

it follows that there exists a perspective mapping $S$ of $L\left(\theta, g^{\prime}\right)$ on $L(\theta, g)$. Now set $h_{0}=h$ and define $h_{n}{ }^{\prime}, h_{n}$, for $n=1,2, \cdots$, by induction on $n$ as follows:

$$
h_{n}^{\prime}=T^{-1}\left(h_{n-1}\right), \quad h_{n}=S\left(h_{n}^{\prime}\right) .
$$

Then $h_{0}, h_{1}, \cdots$ are independent over $\theta$ by the corollary to Lemma 2.6 since

$$
\begin{aligned}
h_{n}\left(h_{n+1}+\cdots+h_{n+p}\right) & =\left(S T^{-1}\right)^{n}\left\{h\left(h_{1}+\cdots+h_{p}\right)\right\} \\
& =\left(S T^{-1}\right)^{n}\left\{h g\left(h_{1}+\cdots+h_{p}\right)\right\} \\
& =\left(S T^{-1}\right)^{n}(\theta)=\theta .
\end{aligned}
$$

Since $h_{n-1} \sim h_{n}{ }^{\prime}, h_{n}{ }^{\prime} \sim h_{n}$, and $h_{n-1} h_{n}=\theta \leqq h_{n}{ }^{\prime}$, Lemma 4.5 shows that $h_{n-1} \sim h_{n}$. Now Lemma 4.3 gives $h=h_{0}=\theta$ as required. 
(V) To complete the proof of Theorem 5.1 we need only show that the $a_{0}=[a-a b]=[a-c]$ defined in (II) above could be chosen in such a way that $c \sum_{n=1}^{\infty} b_{n}{ }^{\prime}=\theta$ will hold in (III) above. We first note that if we set

$$
v_{1}=T^{-1}(c), v_{2}=T^{-1}\left(v_{1} c\right), \cdots, v_{n+1}=T^{-1}\left(v_{n} c\right), \cdots,
$$

then it is sufficient to choose $a_{0}$ so that $a=a_{0} \oplus c$ and

$$
v_{n} c=\left(v_{n}+a_{0}\right) c,
$$

for $n=1,2, \cdots$. For if $a_{0}$ is so chosen, then

$$
\begin{aligned}
& \theta \leqq b_{n+1}^{\prime}\left(c+b_{1}^{\prime}+b_{2}^{\prime}+\cdots+b_{n}^{\prime}\right) \\
& =T\left\{b_{n(n+1)}^{\prime}\left(T^{-1}(c)+a_{1}^{\prime}+b_{12}^{\prime}+\cdots+b_{(n-1) n}^{\prime}\right)\right\} \\
& \leqq T\left\{b_{n(n+1)}^{\prime} c\left(v_{1}+a_{0}+b_{12}^{\prime}+\cdots+b_{(n-1) n}^{\prime}\right)\right\} \\
& =T\left\{b_{n(n+1)}^{\prime}\left(c\left(v_{1}+a_{0}\right)+b_{12}^{\prime}+\cdots+b_{(n-1) n}^{\prime}\right)\right\} \\
& =T\left\{b_{n(n+1)}^{\prime}\left(v_{1} c+b_{12}^{\prime}+\cdots+b_{(n-1) n}^{\prime}\right)\right\} \\
& \leqq T^{2}\left\{b_{(n-1) n(n+1)}^{\prime}\left(v_{2}+a_{0}+b_{123}^{\prime}+\cdots+b_{(n-2)(n-1) n}^{\prime}\right)\right\} \\
& \leqq T^{2}\left\{b_{(n-1) n(n+1)}^{\prime}\left(v_{2} c+b_{123}^{\prime}+\cdots+b_{(n-2)(n-1) n}^{\prime}\right)\right\} \\
& \leqq \cdots \leqq T^{n}\left(b_{12}^{\prime} \cdots(n+1) v_{n} c\right) \leqq b_{n+1}^{\prime} c=\theta \text {; }
\end{aligned}
$$

hence

$$
b_{n+1}^{\prime}\left(c+b_{1}^{\prime}+b_{2}^{\prime}+\cdots+b_{n}^{\prime}\right)=\theta,
$$

for $n=1,2, \cdots$. By Lemma 2.6, $c, b_{1}{ }^{\prime}, b_{2}{ }^{\prime}, \cdots$ are independent over $\theta$; hence $c \sum_{n=1}^{\infty} b_{n}{ }^{\prime}=\theta$ as required.

Thus we have only to construct an $a_{0}$ such that

$$
a=a_{0} \oplus c, \quad v_{n} c=\left(v_{n}+a_{0}\right) c,
$$

for $n=1,2, \cdots$. Apply Lemma 5.1 to $v_{1} \geqq v_{2} \geqq \cdots$ and $c$, and obtain $\bar{I}, I_{n}$, $n=1,2, \cdots$, as in the proof of Lemma 5.1. Then $v_{n}=v_{n} c \oplus \bar{I} \oplus \sum_{m=n}^{\infty} I_{m}$. Let $u=\left[a-\left(v_{1}+c\right)\right]$. Then

$$
u\left(I+\sum_{m=1}^{\infty} I_{m}\right)=u v_{1}\left(I+\sum_{m=1}^{\infty} I_{m}\right)=\theta
$$

and we can set

$$
a_{0}=\bar{I} \oplus \sum_{m=1}^{\infty}\left(\oplus I_{m}\right) \oplus u .
$$

This $a_{0}$ satisfies our requirements, for

$$
a_{0}+c=c+\bar{I}+\sum_{m=1}^{\infty} I_{m}+u=c+v_{1}+u=a,
$$


Hence $a_{0} \oplus c=a$. And

$$
\begin{aligned}
a_{0} c=a_{0}\left(v_{1}+c\right) c & =\left(I+\sum_{m=1}^{\infty} I_{m}\right) c \\
& =\left(I+\sum_{m=1}^{\infty} I_{m}\right) v_{1} c=\theta .
\end{aligned}
$$

$$
\begin{aligned}
\left(v_{n}+a_{0}\right) c & =\left(v_{n} c+I+\sum_{m=1}^{\infty} I_{m}+u\right) c \\
& =\left(v_{n} c+a_{0}\right) c \\
& =v_{n} c+a_{0} c=v_{n} c+\theta=v_{n} c .
\end{aligned}
$$

This completes the proof of Theorem 5.1.

6. Additivity and continuity properties of perspectivity. We prove the following lemma:

LEMMA 6.1. If $\theta$ is defined and if

$$
a=a_{1} \oplus a_{1}^{\prime}=a_{2} \oplus a_{2}^{\prime},
$$

then $a_{1} \sim a_{2}$ implies $a_{1}^{\prime} \sim a_{2}^{\prime}$.

Proof. The perspectivity $a_{1} \sim a_{2}$ implies, by Lemma 3.1 , the existence of an $x$ for which

$$
\begin{aligned}
a_{1}+x & =a_{2}+x=a_{1}+a_{2}, \\
a_{1} x & =a_{2} x=\theta .
\end{aligned}
$$

Let $c=\left[a-\left(a_{1}+a_{2}\right)\right]$. Then $a_{1}^{\prime}$ is perspective to $(x+c)$ with axis $a_{1}$, for we have the relation

$$
\begin{aligned}
a_{1}^{\prime}+a_{1} & =a=c+\left(a_{1}+a_{2}\right)=c+\left(a_{1}+x\right) \\
& =(x+c)+a_{1},
\end{aligned}
$$

hence relation (i) holds; and

$$
\begin{aligned}
a_{1}^{\prime} a_{1} & =\theta=x a_{1}=(x+\theta) a_{1}=\left\{x+\left(a_{1}+a_{2}\right) c\right\} a_{1} \\
& =\left\{x+\left(a_{1}+x\right) c\right\} a_{1}=(x+c) a_{1},
\end{aligned}
$$

hence relation (ii) holds.

Similarly $a_{2}^{\prime}$ is perspective to $(x+c)$. Theorem 5.1 then proves that $a_{1}^{\prime} \sim a_{2}^{\prime}$ as required.

LEMMA 6.2. If $\theta$ is defined, and if

$$
a=a_{1} \oplus a_{1}^{\prime}, \quad b=b_{1} \oplus b_{1}^{\prime},
$$

then $a \sim b, a_{1} \sim b_{1}$ together imply $a_{1}^{\prime} \sim b_{1}^{\prime}$. 
Proof. Let $T$ be a perspective mapping of $L(\theta, a)$ on $L(\theta, b)$, and let $u=T\left(a_{1}\right), v=T\left(a_{1}^{\prime}\right)$; then $a_{1} \sim u, a_{1}^{\prime} \sim v$, and $b=u \oplus v$. Since $a_{1} \sim b_{1}$, Theorem 5.1 gives $u \sim b_{1}$ and Lemma 6.1 gives $v \sim b_{1}{ }^{\prime}$. Since $a_{1}^{\prime} \sim v$ and $v \sim b_{1}^{\prime}$, Theorem 5.1 gives $a_{1}^{\prime} \sim b_{1}{ }^{\prime}$.

LEMMA 6.3. $a_{1} \sim b_{1}, a_{2} \sim b_{2}, a_{1} a_{2}=b_{1} b_{2}$ together imply $\left(a_{1}+a_{2}\right) \sim\left(b_{1}+b_{2}\right)$.

Proof. Let $\theta=a_{1} a_{2}=b_{1} b_{2}, d=a_{1}+a_{2}+b_{1}+b_{2}$, and define $a^{\prime}=\left[d-\left(a_{1} \oplus a_{2}\right)\right]$, $b^{\prime}=\left[d-\left(b_{1} \oplus b_{2}\right)\right]$. Then

$$
\left(a^{\prime} \oplus a_{2}\right) \oplus a_{1}=d=\left(b^{\prime} \oplus b_{2}\right) \oplus b_{1} .
$$

By Lemma 6.1, $\left(a^{\prime} \oplus a_{2}\right) \sim\left(b^{\prime} \oplus b_{2}\right)$; hence by Lemma $6.2 a^{\prime} \sim b^{\prime}$. Since $a^{\prime} \oplus\left(a_{1} \oplus a_{2}\right)=b^{\prime} \oplus\left(b_{1} \oplus b_{2}\right)$ Lemma 6.1 proves $\left(a_{1}+a_{2}\right) \sim\left(b_{1}+b_{2}\right)$ as required.

LEMMA 6.4. If $\theta$ is defined, and if $a_{1}, \cdots, a_{p}$ and $b_{1}, \cdots, b_{p}$ are two sets of elements, each independent over $\theta$, with $a_{r} \sim b_{r}$ for $r=1, \cdots, p$, where $p=1,2, \cdots$, then

$$
\left(\sum_{r=1}^{p}\left(\oplus a_{r}\right)\right) \sim\left(\sum_{r=1}^{p}\left(\oplus b_{r}\right)\right) .
$$

Proof. Suppose the lemma established for $p=n$ for some fixed $n=1,2, \cdots$. Then

$$
\left(a_{1} \oplus \cdots \oplus a_{n}\right) \sim\left(b_{1} \oplus \cdots \oplus b_{n}\right), \quad a_{n+1} \sim b_{n+1}
$$

imply, by Lemma $6.3,\left(a_{1}+\cdots+a_{n}+a_{n+1}\right) \sim\left(b_{1}+\cdots+b_{n}+b_{n+1}\right)$; and the lemma will hold for $p=n+1$. Since the lemma is trivially true for $p=1$, it holds, by induction, for all $p=1,2, \cdots$.

We now define a relation $a \propto b$ as follows:

Definition 6.1. $a \propto b$ if $a \sim b_{1}$ for some $b_{1} \leqq b$.

LEMMA 6.5. (I) $a \leqq b$ implies $a \propto b$.

(II) $a \leqq b, b \propto c$ together imply $a \propto c$.

(III) $a \propto b, b \propto c$ together imply $a \propto c$.

(IV) $a \propto b, b \propto a$ together imply $a \sim b$.

Proof. (I) follows from $a \sim a$.

(II): Let $\theta=a c$, and let $T$ be a perspective mapping of $L(\theta, b)$ on $L(\theta, c)$. Then $T(a)$ is defined, $a \sim T(a)$, and $T(a) \leqq c$. Hence $a \propto c$.

(III) $: a \propto b$ means $a \sim b_{1}$ for some $b_{1} \leqq b$. Since $b \propto c$, (II) gives $b_{1} \sim c_{3}$ for some $c_{1} \leqq c$. Theorem 5.1 then gives $a \sim c_{1}$. Hence $a \propto c$ as required.

(IV): $a \propto b$ means $a \sim b_{1}$ for some $b_{1} \leqq b$. If $b \propto a$, then $b \sim a_{1}$ for some $a_{1} \leqq a$, and $b_{1} \sim a_{2}$ for some $a_{2} \leqq a_{1}$, by (II). Then, by Theorem 5.1, $a \sim a_{2}$; and, 
since $a_{2} \leqq a$, Lemma 4.4 implies $a_{2}=a$. Since $a_{2} \leqq a_{1} \leqq a$, we have $a=a_{1} \sim b$; that is $a \sim b$ as required.

LEMMA 6.6. If $c \propto a$ and $d=c a$, there exists an element $a_{1}$ with $d \leqq a_{1} \leqq a$ and $c \sim a_{1}$.

Proof. $c \propto a$ means $c \sim a_{2}$ for some $a_{2} \leqq a$. Let $\theta=a_{2} d$; then $\theta \leqq c a_{2}$, and there exists, by Lemmas 3.1 and 3.2, a perspective mapping $T$ of $L(\theta, c)$ on $L\left(\theta, a_{2}\right)$. Define $c^{\prime}=[c-d]$ and $\bar{c}^{\prime}=T\left(c^{\prime}\right)$; then $\bar{c}^{\prime} \leqq a_{2} \leqq a, c^{\prime} d=\theta, c^{\prime} \sim \bar{c}^{\prime}$, and $\bar{c}^{\prime} d=\bar{c}^{\prime} a_{2} d$ $=\theta$. By Lemma 6.3,

$$
c=\left(c^{\prime} \oplus d\right) \sim\left(\bar{c}^{\prime} \oplus d\right) \leqq a,
$$

and $a_{1}=\bar{c}^{\prime} \oplus d$ satisfies all the requirements of the lemma.

Lемма 6.7. If $\theta$ is defined and if $a \oplus a_{1} \geqq a^{\prime} \oplus a_{2}$, then $a \sim a^{\prime}$ implies $a_{2} \propto a_{1}$.

Proof. Let $v=\left[\left(a \oplus a_{1}\right)-\left(a^{\prime} \oplus a_{2}\right)\right]$. Then $a \oplus a_{1}=a^{\prime} \oplus a_{2} \oplus v$, and Lemma 6.1 implies $a_{1} \sim\left(a_{2} \oplus v\right)$; thus $a_{2} \propto a_{1}$ as required.

LEMMA 6.8. If $\theta$ is defined and if $a \oplus a_{2} \propto a \oplus a_{1}$, then $a_{2} \propto a_{1}$.

Proof. By Lemma 6.6 (with the $\theta$ of the present lemma in the place of the $d$ of Lemma 6.6) $\left(a \oplus a_{2}\right) \sim u$, where $\theta \leqq u \leqq\left(a+a_{1}\right)$. Let $T$ be a perspective mapping of $L\left(\theta, a+a_{2}\right)$ on $L(\theta, u)$, and let $\bar{a}=T(a)$ and $\bar{a}_{2}=T\left(a_{2}\right)$; then $a \oplus a_{1} \geqq \bar{a} \oplus \bar{a}_{2}$ and $a \sim \bar{a}$. By Lemma 6.7, $a_{2} \propto a_{1}$, and since $\bar{a}_{2} \sim a_{2}$, Lemma 6.5 (III) implies $a_{2} \propto a_{1}$, which proves the lemma.

Leмma 6.9. If $\theta$ is defined, and if

$$
u \oplus v_{1} \oplus \cdots \oplus v_{p} \propto a,
$$

where $u \leqq a$ and $\left(v_{1} \oplus \cdots \oplus v_{p}\right) a=\theta,(p=1,2, \cdots)$, then there exist elements $v_{1}^{\prime}, \cdots, v_{p}^{\prime}$ with $v_{r} \sim v_{r}^{\prime},(r=1, \cdots, p), u, v_{1}, \cdots, v_{p}, v_{1}^{\prime}, \cdots, v_{p}^{\prime}$ independent over $\theta$, and $u \oplus v_{1}^{\prime} \oplus \cdots \oplus v_{p}^{\prime} \leqq a$.

Proof. Define $a_{1}=[a-u]$; then

$$
a=u \oplus a_{1}, u \oplus\left(v_{1} \oplus \cdots \oplus v_{p}\right) \propto u \oplus a_{1},
$$

and Lemma 6.8 implies $\left(v_{1} \oplus \cdots \oplus v_{p}\right) \propto a_{1}$. Thus there exists, by Lemma 6.6, a perspective mapping $T$ of $L\left(\theta,\left(v_{1} \oplus \cdots \oplus v_{p}\right)\right)$ on $L\left(\theta, a_{2}\right)$ for some $a_{2}$ satisfying $\theta \leqq a_{2} \leqq a_{1}$. Let $v_{r}^{\prime}=T\left(v_{r}\right)$ for $r=1, \cdots, p$. Then $v_{r} \sim v_{r}^{\prime}$, for $r=1, \cdots, p ; \sum_{r=1}^{p}\left(\oplus v_{r}^{\prime}\right) \leqq a_{2} \leqq a_{1} \leqq a ;$ and $v_{r}^{\prime},(r=1, \cdots, p)$, are independent over $\theta$. Since

$$
\begin{aligned}
\left(\sum_{r=1}^{p}\left(\oplus v_{r}^{\prime}\right)\right)\left(u \oplus \sum_{r=1}^{p}\left(\oplus v_{r}\right)\right) & =\left(\sum_{r=1}^{p}\left(\oplus v_{r}^{\prime}\right)\right) a_{1} a\left(u \oplus \sum_{r=1}^{p}\left(\oplus v_{r}\right)\right) \\
& =\left(\sum_{r=1}^{p}\left(\oplus v_{r}^{\prime}\right)\right) a_{1} u=\theta,
\end{aligned}
$$


$u, v_{1}, \cdots, v_{p}, v_{1}^{\prime}, \cdots, v_{p}^{\prime}$ are independent over $\theta$ by Lemma 2.10. Thus $v_{1}^{\prime}, \cdots, v_{p}^{\prime}$ satisfy all the requirements of the lemma.

LEMMA 6.10. Let $a_{1} \geqq a_{2} \geqq \cdots$ be an infinite set of elements, and let $c$ satisfy $\theta \leqq c \leqq a_{1}$, where $\theta=\prod_{n} a_{n}$. Suppose further that $c \propto a_{n}$ for $n=1,2, \cdot \cdot \cdot$. Then there exists an element $c^{\prime} \leqq a_{1}$ such that $c \sim c^{\prime}, c c^{\prime}=\theta$, and $\left(c \oplus c^{\prime}\right) \propto a_{n}$ for $n=1,2, \cdots$.

Proof. Define $c_{t}=\left[c a_{t}-c a_{t+1}\right]$ for $t=1,2, \cdots ;$ then $c a_{t}=c a_{t+1} \oplus c_{t}$, and

$$
\begin{aligned}
c=c a_{1} & =\sum_{t=1}^{\infty}\left(\oplus c_{t}\right) \oplus \prod_{t=1}^{\infty}\left(c a_{t}\right) \\
& =\sum_{t=1}^{\infty}\left(\oplus c_{t}\right)+\theta=\sum_{t=1}^{\infty}\left(\oplus c_{t}\right) .
\end{aligned}
$$

Suppose that $c_{r_{1} r_{2}} \cdots r_{n} t$ have been defined, for all $1 \leqq r_{1}<r_{2}<\cdots<r_{n}<t<\infty$. with $1 \leqq r_{n}<p$ for some fixed $p=1,2, \cdots$ ( $n$ taking all values possible), in such a way that the following conditions are satisfied:

$(\alpha)_{p} c_{t}, t=1,2, \cdots ; c_{r_{1} r_{2}} \cdots r_{n} t, 1 \leqq r_{1}<r_{2}<\cdots<r_{n}<t$, for $r_{n}<p$, are independent over $\theta$,

$(\beta)_{p}$ If we set

$$
c_{r_{1} r_{2}}^{\prime} \cdots r_{n}=\sum_{t=r_{n+1}}^{\infty}\left(\oplus c_{r_{1} r_{2} \cdots r_{n} t}\right)
$$

then

$$
c_{r_{1} r_{2}} \cdots r_{n} t=\left[c_{r_{1} r_{2} \cdots r_{n}}^{\prime} a_{t}-c_{r_{1} r_{2} \cdots r_{n}}^{\prime} a_{t+1}\right]
$$

and

$$
\begin{gathered}
c_{r_{1} r_{2} \cdots r_{n}} \sim c_{r_{1} r_{2} \cdots r_{n}}^{\prime}, \quad 1 \leqq r_{1}<r_{2}<\cdots<r_{n}<t<\infty, r_{n}<p . \\
\quad c \sim \sum_{t=p}^{\infty}\left(\oplus c_{t}\right) \oplus \sum_{t=p}^{\infty}\left(\oplus c_{r_{1} r_{2} \cdots r_{n} t}\right),
\end{gathered}
$$

where in the last summation $r_{1}, r_{2}, \cdots, r_{n}$ take on all possible values with $r_{n}<p$.

Then $c \propto a_{p+1}$, and

$$
\begin{aligned}
c \sim\left(\sum_{t=p+1}^{\infty}\left(\oplus c_{t}\right) \oplus \sum_{t=p+1, r_{n}<p}^{\infty}\left(\oplus c_{r_{1} r_{2} \ldots r_{n} t}\right)\right) \\
\oplus\left(c_{p} \oplus \sum_{r_{n}<p}\left(\oplus c_{r_{1} r_{2} \ldots r_{n} p}\right)\right)
\end{aligned}
$$


and Lemma 6.9 secures the existence of elements $c_{p}^{\prime}, c_{r_{1} r_{2} \cdots r_{n} p}^{\prime},\left(1 \leqq r_{1}<r_{2}\right.$ $\left.<\cdots<r_{n}<p\right)$, such that $\theta \leqq c_{p}^{\prime} \leqq a_{p+1}, \theta \leqq c_{r_{1} r_{2} \cdots r_{n} p} \leqq a_{p+1}, c_{p} \sim c_{p}^{\prime}, c_{r_{1} r_{2} \cdots r_{n} p}$ $\sim c_{r_{1} r_{2} \cdots r_{n} p}^{\prime}$, and $c_{t},(t=1,2, \cdots), c_{r_{1} r_{2} \cdots r_{n} t},\left(1 \leqq r_{1}<r_{2}<\cdots<r_{n}<t<\infty\right.$, $\left.r_{n}<p\right), c_{p}^{\prime}, c_{r_{1} r_{2} \cdots r_{n} p}^{\prime},\left(1 \leqq r_{1}<r_{2}<\cdots<r_{n}<p\right)$, are independent over $\theta$. Hence if we define $c_{p t}=\left[c_{p}^{\prime} a_{t}-c_{p}^{\prime} a_{t+1}\right]$ and $c_{r_{1} r_{2}} \ldots r_{n} p t=\left[c_{r_{1} r_{2}}^{\prime} \cdots r_{n} p a_{t}-c_{r_{1} r_{2}}^{\prime} \ldots r_{n} p a_{t+1}\right]$, then $(\alpha)_{p+1},(\beta)_{p+1}$, and $(\gamma)_{p+1}$ will be satisfied. Since $(\alpha)_{1},(\beta)_{1}$, and $(\gamma)_{1}$ are trivially satisfied, it follows that we can define the $c_{r_{1} r_{2} \ldots r_{n} t}$, (for all $\left.1 \leqq r_{1}<r_{2}<\cdots<r_{n}<t<\infty\right)$, so that $(\alpha)_{p},(\beta)_{p}$, and $(\gamma)_{p}$ are satisfied for all $p=1,2, \cdots$. Then $c_{1}, c_{2}, \cdots, c_{1}{ }^{\prime}, c_{2}^{\prime}, \cdots$ are independent over $\theta$, and $c_{n} \sim c_{n}{ }^{\prime}$ for $n=1,2, \cdots$. Lemmas 2.3 and 4.2 now imply that $c=\sum_{n-1}^{\infty}\left(\oplus c_{n}\right)$ $\sim \sum_{n=1}^{\infty}\left(\oplus c_{n}^{\prime}\right)$. If we set $c^{\prime}=\sum_{n-1}^{\infty}\left(\oplus c_{n}^{\prime}\right)$, we will have $c \sim c^{\prime}, c c^{\prime}=\theta$, and $\left(c \oplus c^{\prime}\right) \leqq a_{1}$.

Finally, since $c \propto a_{p}$, we have $c \sim c^{p}$ for some $c^{p}$ with $\theta \leqq c^{p} \leqq a_{p}$ by Lemma 6.6. Applying the reasoning of the preceding paragraph to $c^{p}$ and $a_{p} \geqq a_{p+1} \geqq \cdots$ we obtain $c^{p^{\prime}}$ such that $c^{p} \sim c^{p^{\prime}}$ and $\left(c^{p} \oplus c^{p^{\prime}}\right) \leqq a_{p}$. Then $c^{\prime} \sim c, c \sim c^{p}, c^{p} \sim c^{p^{\prime}}$, imply, by Theorem 5.1, $c^{\prime} \sim c^{p^{\prime}}$ and hence, by Lemma 6.3, $\left(c \oplus c^{\prime}\right) \sim\left(c^{p} \oplus c^{p^{\prime}}\right)$. Lemma 6.5 (III) now implies that $\left(c \oplus c^{\prime}\right) \propto a_{p}$ for all $p=1,2, \cdots$, and $c^{\prime}$ satisfies all the requirements of the lemma.

\section{LEMMA 6.11. The hypotheses of Lemma 6.10 imply $c=\theta$.}

Proof. Suppose that $\bar{c}_{1}, \bar{c}_{2}, \cdots, \bar{c}_{2}$ p have been defined for some fixed $p=0,1, \cdots$ in such a way that, if we write $c_{(p)}$ for $\bar{c}_{1}+\cdots+\bar{c}_{p}$, then

$(\lambda)_{p} c \sim \bar{c}_{r}$ for $r=1, \cdots, 2^{p}$

$(\mu)_{p} \bar{c}_{r}, r=1, \cdots, 2^{p}$, are independent over $\theta$;

$(\nu)_{p} c_{(p)} \leqq a_{1}$ and $c_{(p)} \propto a_{n}$ for $n=1,2, \cdots$.

Then there exists, by Lemma 6.10, an element $c^{\prime} \leqq a_{1}$ such that $c_{(p)} \sim c^{\prime}$, $c_{(p)} c^{\prime}=\theta$, and $\left(c_{(p)} \oplus c^{\prime}\right) \propto a_{n}$ for $n=1,2, \cdots$. Let $T$ be a perspective mapping of $L\left(\theta, c_{(p)}\right)$ on $L\left(\theta, c^{\prime}\right)$, and let $\bar{c}_{2^{p}+r}=T\left(\bar{c}_{r}\right)$ for $r=1, \cdots, 2^{p}$. Then $(\lambda)_{p+1}$, $(\mu)_{p+1}$, and $(\nu)_{p+1}$ will be satisfied. Since we can define $\bar{c}_{1}=c$ to satisfy $(\lambda)_{0}$, $(\mu)_{0}$, and $(\nu)_{0}$, it follows that we can define, by induction on $p$, an infinite sequence $\bar{c}_{n},(n=1,2, \cdots)$, satisfying $(\lambda)_{p},(\mu)_{p}$, and $(\nu)_{p}$, for all $p=0,1, \cdots$. Then we have $\bar{c}_{1}, \bar{c}_{2}, \ldots$ independent over $\theta$ and $\bar{c}_{n} \sim \bar{c}_{n+1}$ (by Theorem 5.1 since $\left.\bar{c}_{n} \sim c, c \sim \bar{c}_{n+1}\right)$; hence $\bar{c}_{1}=\theta$ by Lemma 4.3. Since $c=\bar{c}_{1}$ we have $c=\theta$. This proves the lemma.

LEMMA 6.12. Without the condition $c \leqq a_{1}$, the remaining hypotheses of Lemma 6.10 imply $c=\theta$.

Proof. $\theta \leqq c a_{1}, c \propto a_{1}$, imply, by Lemma 6.6, the existence of a $c_{1}$ with $\theta \leqq c_{1} \leqq a_{1}$, and $c \sim c_{1}$. By Lemma 6.5 (III), $c_{1}$ and $a_{1} \geqq a_{2} \geqq \cdots$ satisfy the 
hypotheses of Lemma 6.10; hence Lemma 6.11 implies $c_{1}=\theta$. Since $c \sim c_{1}=\theta$ and $\theta \leqq c$, Lemma 4.4 gives $c=\theta$. This proves the lemma.

LeMma 6.13. If $\theta$ is defined and $c \propto(a \oplus b)$, there exists a decomposition $c=c_{1} \oplus c_{2}$ with $c_{1} \propto a, c_{2} \propto b$.

Proof. By Lemma 6.6, $\sim \sim u, \theta \leqq u \leqq(a \oplus b)$. Let $u_{1}=a u$, define $u_{1}^{\prime}=\left[u-u_{1}\right]$, and let $u_{2}=\left(a+u_{1}^{\prime}\right) b$; then $u=u_{1} \oplus u_{1}^{\prime}$, and $u_{2} \sim u_{1}^{\prime}$ with axis $a$, for

$$
u_{1}^{\prime}+a=\left(u_{1}^{\prime}+a\right)(b+a)=\left(a+u_{1}^{\prime}\right) b+a=u_{2}+a,
$$

hence (i) is satisfied; and

$$
u_{1}^{\prime} a=u_{1}^{\prime} u a=u_{1}^{\prime} u_{1}=\theta=u_{2} b a=u_{2} a,
$$

hence (ii) is satisfied.

Now let $c_{1}=T^{-1}\left(u_{1}\right)$ and $c_{2}=T^{-1}\left(u_{1}^{\prime}\right)$; then $c=c_{1} \oplus c_{2}, c_{1} \sim u_{1} \leqq a$, and $c_{2} \sim u_{2} \leqq b$ (by Theorem 5.1, since $c_{2} \sim u_{1}^{\prime}$ and $u_{1}^{\prime} \sim u_{2}$ ). This proves the lemma.

LEMMA 6.14. If $a_{1} \geqq a_{2} \geqq \cdots$ and $c \propto a_{n}$ for $n=1,2, \cdots$, then $c \propto \prod_{n} a_{n}$.

Proof. Let $\bar{a}=\prod_{n} a_{n}, \theta=c \bar{a}$. Apply Lemma 5.1 to $\bar{a}$ and $a_{1} \geqq a_{2} \geqq \cdots$ to obtain $a_{n}=a_{n}^{\prime} \oplus \bar{a}$ with $a_{1}^{\prime} \geqq a_{2}^{\prime} \geqq \cdots$. Then $\prod_{n} a_{n}{ }^{\prime}=\left(\prod_{n} a_{n}{ }^{\prime}\right) \bar{a} a_{1}^{\prime}=\theta$.

Let $c_{0}=c$ and $\bar{a}_{0}=\bar{a}$. Suppose $c_{r}, c_{r}^{\prime}, \bar{a}_{r}, \bar{a}_{r}^{\prime}$, have been defined for $1 \leqq r<p$ and for some $p=1,2, \cdots$ in such a way that the following conditions are satisfied:

$(\alpha)_{p} c_{r-1}=c_{r} \oplus c_{r}^{\prime}, \bar{a}_{r-1}=\bar{a}_{r} \oplus \bar{a}_{r}^{\prime}, c_{r}^{\prime} \sim \bar{a}_{r}^{\prime}$, for $1 \leqq r<p$.

$(\beta)_{p} c_{p-1} \propto a_{p-1}^{\prime},(p>1)$, and $c_{p-1} \propto\left(a_{n}^{\prime}+\bar{a}_{p-1}\right)$, for $n=1,2, \cdots$.

Then, since $c_{p-1} \propto\left(a_{p}^{\prime}+\bar{a}_{p}\right)$, we can define $c_{p}, c_{p}^{\prime}, \bar{a}_{p}^{\prime}$, by Lemma 6.13 , so that

$$
c_{p-1}=c_{p} \oplus c_{p}^{\prime}, \quad c_{p} \propto a_{p}^{\prime}, \quad c_{p}^{\prime} \sim \bar{a}_{p}^{\prime} \leqq \bar{a}_{p-1} .
$$

Now define $\bar{a}_{p}=\left[\bar{a}_{p-1}-\bar{a}_{p}{ }^{\prime}\right]$. Then, by the use of Lemma 6.8,

$$
\bar{a}_{p-1}=\bar{a}_{p} \oplus \bar{a}_{p}^{\prime}, \quad c_{p} \propto\left(a_{n}^{\prime} \oplus \bar{a}_{p}\right), \quad \text { for } \quad n=1,2, \cdots .
$$

Thus $(\alpha)_{p+1},(\beta)_{p+1}$ are satisfied. Since $(\alpha)_{1}$ and $(\beta)_{1}$ are satisfied by $c_{0}, \bar{a}_{0}$, it follows that we can define by induction $c_{r}, c_{r}^{\prime}, \bar{a}_{r}, \bar{a}_{r}^{\prime}$, for $r=1,2, \cdots$, to satisfy $(\alpha)_{p}$ and $(\beta)_{p}$ for all $p=1,2, \cdots$.

By Lemma 2.11

Since

$$
c=\sum_{n=1}^{\infty}\left(\oplus c_{n}^{\prime}\right) \oplus \prod_{n=1}^{\infty} c_{n}, \quad \bar{a}=\sum_{n=1}^{\infty}\left(\oplus \bar{a}_{n}^{\prime}\right) \oplus \prod_{n=1}^{\infty} \bar{a}_{n} .
$$

$$
\prod_{n=1}^{\infty} a_{n}^{\prime}=\theta \leqq \prod_{n=1}^{\infty} c_{n} \leqq c_{r} \propto a_{r}^{\prime} \quad \text { for } \quad r=1,2, \cdots
$$


Lemma 6.12 implies $\prod_{n=1}^{\infty} c_{n}=\theta$. Hence $c=\sum_{n=1}^{\infty}\left(\oplus c_{n}^{\prime}\right)$. Since

$$
c\left(\sum_{n=1}^{\infty}\left(\oplus \bar{a}_{n}^{\prime}\right)\right)=c \bar{a}\left(\sum_{n=1}^{\infty}\left(\oplus \bar{a}_{n}^{\prime}\right)\right)=\theta,
$$

Lemma 2.10 implies $c_{n}{ }^{\prime}, a_{n}{ }^{\prime},(n=1,2, \cdots)$, are independent. Lemmas 2.3 and 4.2 now give

$$
c=\sum_{n=1}^{\infty}\left(\oplus c_{n}^{\prime}\right) \sim \sum_{n=1}^{\infty}\left(\oplus \bar{a}_{n}\right) \leqq \bar{a}=\prod_{n=1}^{\infty} a_{n} .
$$

Hence $\dot{c} \propto \prod_{n} a_{n}$, which proves the lemma.

LEMma 6.15. If $\theta$ is defined, and if

$$
a \oplus a^{\prime}=b \oplus b^{\prime},
$$

then $b \propto a$ implies $a^{\prime} \propto b^{\prime}$.

Proof. Suppose $b \sim a_{1} \leqq a$, and define $a_{1}^{\prime}=\left[a-a_{1}\right]$. Then $a_{1} \oplus\left(a_{1}^{\prime} \oplus a^{\prime}\right)$ $=b \oplus b^{\prime}$, and Lemma 6.2 implies $\left(a_{1}^{\prime} \oplus a^{\prime}\right) \sim b^{\prime}$; hence by Lemma 6.5 (II) $a^{\prime} \propto b^{\prime}$.

LEMma 6.16. If $a_{1} \leqq a_{2} \leqq \cdots$ and if $a_{n} \propto c$ for $n=1,2, \cdots$, then $\sum_{n} a_{n} \propto c$.

Proof. Let $a_{1} c=\theta$, and define $u_{1}=a_{1}, u_{n}=\left[a_{n}-a_{n-1}\right]$ for $n=2,3, \cdots$; then $a_{n}=\sum_{r-1}^{n} u_{r},(n=1,2, \cdots)$, and $u_{1}, u_{2}, \cdots$ are independent over $\theta$ by Lemma 2.6, since $u_{n+1}\left(u_{1}+\cdots+u_{n}\right)=\left[a_{n+1}-a_{n}\right] a_{n}=\theta$ for $n=1,2, \cdots$. Set $b_{n}=\sum_{r=n+1}^{\infty}\left(\oplus u_{r}\right)$ for $n=0,1, \cdots ;$ then $b_{0} \geqq b_{1} \geqq \cdots$, and

$$
b_{0}=\sum_{r=1}^{\infty}\left(\oplus u_{r}\right)=a_{n} \oplus b_{n}, \quad n=1,2, \cdots .
$$

Hence $b_{0} \geqq \sum_{n=1}^{\infty} a_{n}$. Since $u_{n} \leqq a_{n}$ we also have $b_{0}=\sum_{n=1}^{\infty} u_{n} \leqq \sum_{n=1}^{\infty} a_{n}$; thus $b_{0}=\sum_{n=1}^{\infty} a_{n}$. Now define $c^{\prime}=\left[\left(b_{0}+c\right)-c\right]$ and $b_{0}{ }^{\prime}=\left[\left(b_{0}+c\right)-b_{0}\right]$. Then

$$
c \oplus c^{\prime}=b_{0} \oplus b_{0}^{\prime}=a_{n} \oplus\left(b_{n} \oplus b_{0}^{\prime}\right)
$$

for $n=1,2, \cdots$, and Lemma 6.15 implies $c^{\prime} \propto\left(b_{n} \oplus b_{0}{ }^{\prime}\right)$ for $n=1,2, \cdots$. Applying Lemma 6.14 to $c^{\prime}$ and $\left(b_{1}+b_{0}^{\prime}\right) \geqq\left(b_{2}+b_{0}^{\prime}\right) \geqq \cdots$, we obtain

$$
c^{\prime} \propto \prod_{n}\left(b_{n}+b_{0}^{\prime}\right)=\left(\prod_{n} b_{n}\right)+b_{0}^{\prime}=\theta+b_{0}^{\prime}=b_{0}^{\prime} .
$$

Lemma 6.15 now implies $\sum_{n} a_{n}=b_{0} \propto c$. This proves the lemma.

Definition 6.2. If $a_{1}, a_{2}, \cdots$ is an infinite sequence, we define

$$
\lim \sup a_{n}=\prod_{p}\left(\sum_{n=p}^{\infty} a_{n}\right), \quad \lim \inf a_{n}=\sum_{p}\left(\prod_{n=p}^{\infty} a_{n}\right) .
$$


The sequence is called convergent if $\lim \sup a_{n}=\lim \inf a_{n}$, and for a convergent sequence we define $\lim a_{n}=\lim \sup a_{n}=\lim \inf a_{n}$.

LEMmA 6.17. If $a_{1} \leqq a_{2} \leqq \cdots\left(a_{1} \geqq a_{2} \geqq \cdots\right)$ then $\lim a_{n}$ is defined and is equal to $\sum_{n} a_{n}\left(\prod_{n} a_{n}\right)$.

Proof.

$$
\begin{aligned}
\lim \sup a_{n}=\prod_{p=1}^{\infty}\left(\sum_{n=p}^{\infty} a_{n}\right) & =\prod_{p=1}^{\infty}\left(\sum_{n=1}^{\infty} a_{n}\right)=\sum_{n=1}^{\infty} a_{n} \\
& \left(=\prod_{p=1}^{\infty}\left(a_{p}\right)=\prod_{n} a_{n}\right) ; \\
\lim \inf a_{n}=\sum_{p=1}^{\infty}\left(\prod_{n=p}^{\infty} a_{n}\right)= & \sum_{p=1}^{\infty}\left(a_{p}\right)=\sum_{n} a_{n} \\
( & \left(=\sum_{p=1}^{\infty}\left(\prod_{n=1}^{\infty} a_{n}\right)=\prod_{n=1}^{\infty} a_{n}\right) .
\end{aligned}
$$

Hence $\lim \sup a_{n}=\lim \inf a_{n}=\sum_{n} a_{n}\left(=\prod_{n} a_{n}\right)$ and the lemma follows from Definition 6.2.

Theorem 6.1. Continuity of PERSPeCtivity. If $a_{1}, a_{2}, \cdots$ and $b_{1}, b_{2}, \cdots$ are convergent sequences with $\lim a_{n}=\bar{a}$ and $\lim b_{n}=\bar{b}$, then $a_{n} \sim b_{n}$ for $n=1,2, \cdots$ implies $\bar{a} \sim \bar{b}$.

Proof. For every fixed $p=1,2, \cdots$ we have

$$
\left(\prod_{n=p}^{\infty} a_{n}\right) \leqq a_{r} \sim b_{r} \leqq\left(\sum_{n=r}^{\infty} b_{n}\right), \quad r=p, p+1, \cdots,
$$

and Lemma 6.14 implies $\left(\prod_{n=p}^{\infty} a_{n}\right) \propto \prod_{r=p}^{\infty}\left(\sum_{n-r}^{\infty} b_{n}\right)=\bar{b}$. Lemma 6.16 gives $\bar{a}=\sum_{p=1}^{\infty}\left(\prod_{n=p}^{\infty} a_{n}\right) \propto \bar{b}$, that is $\bar{a} \propto \bar{b}$. Similarly $\bar{b} \propto \bar{a}$. Then, by Lemma 6.15(IV), $\bar{a} \sim \bar{b}$, which proves the theorem.

CoROLLARY. If $a_{1} \leqq a_{2} \leqq \cdots$ and $b_{1} \leqq b_{2} \leqq \cdots\left(a_{1} \geqq a_{2} \geqq \cdots\right.$ and $b_{1} \geqq b_{2}$ $\geqq \cdots$ ) then $a_{n} \sim b_{n}$ for $n=1,2, \cdots$ implies $\sum_{n} a_{n} \sim \sum_{n} b_{n}\left(\prod_{n} a_{n} \sim \prod_{n} b_{n}\right)$.

Proof. By Lemma 6.17 this is a special case of Theorem 6.1.

Theorem 6.2. AdDItIVITy OF PERSPectivity. If $\theta$ is defined and if $a_{n}$, $1 \leqq n<p$, and $b_{n}, 1 \leqq n<p$, are each independent over $\theta$, where $p$ is finite or infinite, then $a_{n} \sim b_{n}$ for $1 \leqq n<p$ implies

$$
\sum_{n=1}^{p}\left(\oplus a_{n}\right) \sim \sum_{n=1}^{p}\left(\oplus b_{n}\right)
$$


Proof. By Lemma $6.4 \sum_{n-1}^{r}\left(\oplus a_{n}\right) \sim \sum_{n-1}^{r}\left(\oplus b_{n}\right)$ for all $r<p$. If $p$ is finite this proves the theorem, and if $p$ is infinite we have, using the corollary to Theorem 6.1,

$$
\sum_{n=1}^{\infty}\left(\oplus a_{n}\right)=\sum_{r}\left(\sum_{n=1}^{r}\left(\oplus a_{n}\right)\right) \sim \sum_{r}\left(\sum_{n=1}^{r}\left(\oplus b_{n}\right)\right)=\sum_{n=1}^{\infty}\left(\oplus b_{n}\right) .
$$

This proves the theorem.*

* For the special case of an irreducible geometry (finite dimensional or continuous), all the lemmas and theorems of $\$ 6$ are easy consequences of the existence of a dimension function, and conversely, some of them are useful in establishing the existence of the dimension function (see C.G. part 1, chaps. 6 and 7). The notion of a convergent sequence is given in an equivalent form by von Neumann, Proceedings of the National Academy of Sciences, vol. 22 (1936), p. 107 (see the definition of $\lim ^{* *}$ given there).

YALE UNIVERSITY, New Haven, Conn. 